\section{Christoph Bachmann}

\section{Einleitung}

Im Jahre 1977 registrierte die IKS ein Präparat namens Padma 28 und stellte der Herstellerfirma, der Padma AG, die Registrierungsurkunde aus (Kasten 1 und 2, Abb. 1, Tab. 1). Dies war ein unscheinbarer Vorgang, denn auch damals wurden jedes Jahr viele Arzneipräparate zur Registrierung eingereicht. Und trotzdem stellte die Registrierung von Padma 28 eine Novität dar, denn die IKS war die erste westliche Registrierungsbehörde, die diesem Arzneimittel, das auf einer Rezeptur der Tibetischen Medizin beruhte, die Zulassung erteilte. Und damit wurde ein kleines Kapitel Medizingeschichte geschrieben. Denn: In

Tab. 1. Zusammensetzung von Padma 28

\begin{tabular}{lr}
\hline Akeleikraut & $15 \mathrm{mg}$ \\
Baldrianwurzel & $10 \mathrm{mg}$ \\
Calciumsulfat-Hemihydrat & $20 \mathrm{mg}$ \\
D-Campher & $4 \mathrm{mg}$ \\
Eisenhutknollen & $1 \mathrm{mg}$ \\
Gartenlattich & $6 \mathrm{mg}$ \\
Gewürznelken & $12 \mathrm{mg}$ \\
Goldfingerkraut & $15 \mathrm{mg}$ \\
Kaempferia-galanga-Rhizom & $10 \mathrm{mg}$ \\
$\quad$ (indische Gewürzlilie) & $40 \mathrm{mg}$ \\
Indische Kostuswurzel & $40 \mathrm{mg}$ \\
Isländisches Moos & $30 \mathrm{mg}$ \\
Kardamomfrucht & $20 \mathrm{mg}$ \\
Marmelosfrucht & $30 \mathrm{mg}$ \\
Myrobalanenfrucht & $25 \mathrm{mg}$ \\
Nelkenpfeffer & $35 \mathrm{mg}$ \\
Nimbaumfrucht & $5 \mathrm{mg}$ \\
Ringelblumenblüten & $30 \mathrm{mg}$ \\
Rotes Sandelholz & $10 \mathrm{mg}$ \\
Sidakraut & $15 \mathrm{mg}$ \\
Spitzwegerichblätter & $15 \mathrm{mg}$ \\
Süssholzwurzel & $15 \mathrm{mg}$ \\
Vogelknöterichkraut & \\
\hline
\end{tabular}

\title{
40 Jahre Padma 28 - eine vierzigjährige Erfolgsgeschichte
}

Vor 40 Jahren erteilte die damalige Interkantonale Kontrollstelle für Heilmittel (IKS), die Vorgängerin der Swissmedic, als erste westliche Registrierungsbehörde dem tibetischen Vielstoffgemisch Padma 28 die Registrierung. Dieses Präparat vereint Kenntnisse der Tibetischen Medizin mit westlichen Qualitätsvorschriften für die Herstellung und Überprüfung der Wirksamkeit. Zahlreiche Studien bestätigen die Wirksamkeit von Padma 28 bei Durchblutungsstörungen.

den 1970er-Jahren waren komplementärmedizinische Behandlungsmethoden wie Traditionelle Chinesische Medizin (TCM), Klassische Homöopathie usw. zwar schon in den westlichen Ländern bekannt, entsprechende Medikamente hatten aber kaum eine Chance auf Arzneimittelregistrierung. Wie gelang diese Pionierleistung?

\section{Wie alles begann}

An dieser Stelle muss gesagt werden, dass noch vor der Registrierung von Padma 28 ein anderes Präparat der Firma Padma die Registrierung erhielt, nämlich Padma Lax. Dieses Arzneimittel erhielt schon 1970 die Zulassung durch die IKS. Die Firma Padma hatte dieses Anthrachinon-haltige Komplex-Laxativum schon einige Jahre vor Padma 28 zur Registrierung eingereicht - in der Hoffnung, die auch für westliche Medizin «verständlichen» Inhaltsstoffe hätten eher eine Chance zur Genehmigung. Padma Lax enthielt damals und heute immer noch unter anderem Aloe, Faulbaumrinde und Rhabarberwurzel.

\section{7: Padma 28}

Bis zur Registrierung von Padma 28 als nächstes Tibetisches Arzneimittel war es aber ein langer und mühsamer Weg. Die vielen, im Westen zum Teil kaum bekannten pflanzlichen Wirkstoffe stellten die IKS wie
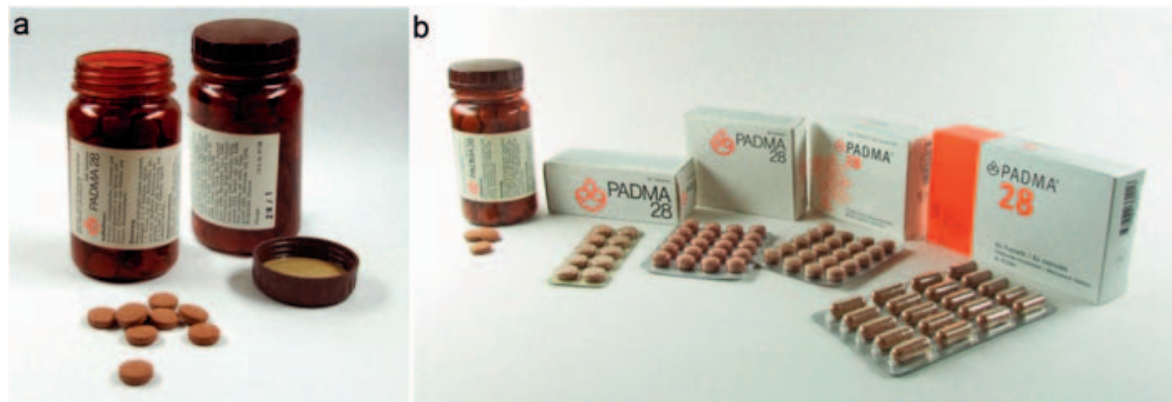

Abb. 1. Padma 28. a 1977: Erste Verpackung von Padma 28 (damals in Tablettenform); b 2009: Wechsel von Tabletten auf Kapseln und neue Verpackung. Dank dieses Wechsels konnten die Hilfsstoffe reduziert werden und die Einnahme wurde erleichtert.

\section{KARGER} (C) 2017 S. Karger GmbH, Freiburg 
Kasten 1. Padma 28

Der Name Padma 28 leitet sich davon ab, dass
sie die 28. Rezeptur in der Rezepturensammlung
der Ärztefamilie Badmajev war, auf der die
Padma-Präparate hauptsächlich basieren.
Padma 28 besteht also nicht aus 28, sondern aus
22 vorwiegend pflanzlichen Wirkstoffen, die
unter anderem folgende chemische Inhaltsstoff-
gruppen enthalten:
- D-Campher: kreislauftonisierend
- Eugenol: antibakteriell und lokal analgetisch
- Flavonoide: entzündungshemmend, antioxida-
tiv, binden freie Metalle
- Gerbstoffe: lokal entzündungshemmend,
antioxidativ
Da es sich um ein komplexes Vielstoffgemisch
handelt, können die einzelnen Wirkstoffe nied-
rig dosiert werden. Diese entfalten untereinan-
der eine synergistische Wirkung und führen zu
einer hohen Verträglichkeit.
Padma 28 (Swissmedic Nr. 58436 ) ist identisch
mit dem Präparat Padmed Circosan (Swiss-
medic Nr. 60131), das von der Grundversicher-
ung erstattet wird.

auch die Firma Padma vor grosse Herausforderungen. Die Registrierungsbehörde verlangte immer wieder neue Unterlagen über die Wirkungen und die Unbedenklichkeit dieser tibetischen Arzneipflanzen. Schliesslich führte aber die Beharrlichkeit zum Erfolg, und die Firma Padma erhielt 1977 die Registrierungsurkunde für ihr Präparat. Vielleicht spielten erste publizierte Studien zu dem Präparat sowie ein gewisser Druck aus der Bevölkerung, die das Präparat durch die Empfehlung von engagierten Fachleuten kennen gelernt hatte, auch eine gewisse Rolle.

Die zugelassenen Indikationen waren damals wie heute Symptome von Durchblutungsstörungen: Durchblutungsstörungen mit Beschwerden wie Kribbeln, Ameisenlaufen, Schwereund Spannungsgefühl in den Beinen und Armen, Einschlafen von Händen und Füssen sowie Wadenkrämpfe.

\section{Studien}

Den Anforderungen der modernen westlichen Medizin entsprechend er-

Kasten 2. Die Firma Padma

Die Firma Padma wurde 1969 gegründet und ist weltweit die einzige Herstellerin von pflanzlichen Rezepturen aus der Tibetischen Medizin und Konstitutionslehre, die nach Schweizer Qualitätsvorschriften produziert. Insofern ist die Firma Padma eine Brückenbauerin zwischen verschiedenen Medizinsystemen und Kulturen.

Der Firmenname Padma soll vor allem an die erste tibetische Ärztefamilie im Westen erinnern. Es handelte sich um den Tibetischen Arzt Sul Tim Badma, der im 19. Jahrhundert im kulturell tibetisch geprägten Burjatien (Sibirien) praktizierte und mit der Übersiedlung nach St. Petersburg den Namen Badmajev annahm.

Padma ist ausserdem ein Sanskrit-Wort für Lotusblume. Damit soll der Verbundenheit mit den Pflanzen Ausdruck verliehen werden.

Nach der Okkupation Tibets durch China im Jahre 1953 gelangten mit dem Dalai Lama auch tibetische Ärzte via Indien in den Westen und eröffneten so neben der Familie von Sul Tim Badma einen zweiten Weg zum Bekanntwerden der Tibetischen Medizin in den westlichen Ländern.

1960 erhielt der Gründer der Padma, Karl Lutz, die Familien-Sammlung der tibetischen Rezepturen der Familie Badmajev. Dies bildete die Grundlage der heutigen Präparate der Firma Padma.

teilte die Firma Padma bald Aufträge für Studien mit Padma 28.

\section{Laborstudie, 1972}

Eine erste Laborstudie aus dem Jahre 1972 befasste sich mit der Thrombozytenaggregation.

\section{Hürlimann, 1978}

Die erste klinische placebokontrollierte Doppelblindstudie wurde 1978 von einem Luzerner Arzt publiziert [1]. Er behandelte Patienten mit peripherer arterieller Verschlusskrankheit (PAVK) im Stadium II während 12 Wochen mit Padma 28 oder mit Placebo und fand folgende Resultate:

- Padma 28 erhöhte die schmerzfreie Gehstrecke signifikant.

- Eine objektive Veränderung der Wadendurchblutung wurde in keiner der beiden Gruppen ermittelt.

- In der Padma 28-Gruppe trat bei nächtlichen Krämpfen, Parästhesien und Restless-legs-Beschwerden eine deutliche Besserung ein.

- Bei Patienten mit PAVK III und IV wurde in der Padma-Gruppe eine Verbesserung der Ruheschmerzen beobachtet.

- Nebenwirkungen traten nicht auf, und Interaktionen mit gleichzeitig verabreichten Medikamenten wie z.B. Antikoagulantien oder Antihypertensiva wurden nicht festgestellt.

\section{Brzosko et al., 1982 und 1983}

Auch in Polen wurde zu Padma 28 geforscht. Die Familie Badmajev floh während der russischen Revolution nach Polen, führte dort die Tradition der Tibetischen Medizin weiter und veröffentliche 1982 und 1983 Berichte über die positive Wirksamkeit von Padma 28 in Anwendungsgebieten ausserhalb von Durchblutungsstörungen wie Hepatitis und entzündliche, degenerative oder neoplastische Gewebeerkrankungen $[2,3]$.

Diese Studien zeigen ein breites Wirkungsspektrum von Padma 28 auf und belegen eine klinische Wirksamkeit in Anwendungsgebieten, die zum Teil auch ausserhalb der heute in der Schweiz zugelassenen Indikationen liegen.

\section{Matzner und Sallon, 1995}

Hinweise zu den vielfältigen Wirkmechanismen gibt auch eine pharmakologische Studie aus Israel, die 1995 publiziert wurde und aufzeigt, dass Padma 28 eine entzündungshemmende Wirksamkeit besitzt [4].

\section{Metaanalyse PAVK, 2006}

Es ist ungewöhnlich für ein pflanzliches Arzneimittel, besonders für ein so komplex zusammengesetztes wie Padma 28, dass genügend qualitativ hochwertige Studien existieren, um eine Metaanalyse durchführen $\mathrm{zu}$ 
können. Die Rohdaten von 5 klinischen Studien zu Padma 28 bei PAVK mit vergleichbaren Zielparametern (Hauptparameter: Verlängerung der maximalen Gehstrecke) wurden von Wissenschaftlern der Universität Zürich zusammengefasst und neu ausgewertet [5]. 274 Patientendaten konnten in die Analyse eingeschlossen werden, und eine klinisch relevante Verbesserung, d.h. eine Gehstreckenverlängerung um $>100$ Meter, trat bei 18,2\% der Patienten der Verum-Gruppe ein, im Vergleich dazu nur bei 2,1\% der Placebo-Patienten. Dies zeigte eine hochsignifikante Überlegenheit für das Verum $(\mathrm{p}<0,001)$.

\section{Übersichtsarbeit klinische Studien} mit Padma 28, 2013

Einen sehr schönen Überblick über die mit Padma 28 durchgeführten klinischen Studien liefert ein Review aus dem Jahre 2013 [6]. Dieser fand 29 klinische Studien (1 Metaanalyse, 21 kontrollierte und 7 offene Studien) und 3 retrospektive Fallserien. Diese umfassten insgesamt 1704 Verum-Patienten, 697 davon Kinder. 333 Patienten waren Placebo-Gruppen zugeteilt. Bei 394 Probanden handelte es sich um unbehandelte oder gesunde Kontrollpatienten. 11 Studien wurden mit Patienten durchgeführt, die an Claudicatio intermittens litten, und wiesen die höchste methodologische Qualität aller Studien auf.

Weitere Studien zeigen die Wirksamkeit von Padma 28 bei anderen vaskulären Erkrankungen als Claudicatio intermittens (6 Studien) sowie bei verschiedenen entzündlichen Beschwerden $(n=12)$, wie z.B. rezidivierende Atemwegsinfekte, Virushepatitis oder Multiple Sklerose.

Die Übersicht zeigt ein sehr günstiges Sicherheitsprofil für Padma 28. Es wurden nur wenige unterwünschte Ereignisse dokumentiert, von denen nur neun erheblich waren und wahrscheinlich im Zusammenhang mit der bestehenden Erkrankung standen. Unerwünschte Ereignisse traten drei- einhalb mal häufiger in der PlaceboGruppe als in der Verum-Gruppe auf. $41 \%$ der in die Studien aufgenommenen Probanden waren Kinder. Auch für diese erwies sich Padma 28 als sehr gut verträglich.

\section{Cochrane Review, 2016}

2016 ist ein Review der Cochrane Collaboration erschienen, der sich mit der Wirksamkeit von Padma 28 bei Claudicatio intermittens befasst [7]. Reviews der Cochrane Collaboration sind der Phytotherapie gegenüber sehr skeptisch eingestellt und ziehen häufig trotz positiver Studienresultate negative Schlussfolgerungen.

Im Fall von Padma 28 bei PAVK stimmen die Autoren trotz der Kritik an einer kleinen Datenmenge und an der Studienqualität grundsätzlich mit den Resultaten der Metaanalyse von Melzer et al. [5] überein. Dass sie dem Präparat eine mögliche Wirksamkeit einräumen sowie eine hohe Sicherheit vermuten, ist im Hinblick auf ihre Beurteilung von Phytotherapeutika allgemein bereits als Auszeichnung zu werten.

\section{Erfahrungsberichte aus Arztpraxen}

Es gibt auch eine Fülle von Erfahrungsberichten zur Anwendung von Padma 28, die in Arztpraxen durchgeführt wurde. Als Beispiel sollen hier zwei Fallberichte aus Schweizer Arztpraxen gekürzt vorgestellt werden, die 2010 publiziert wurden [8].

Bericht: Dr. med. Eva Dytrich, Bern Patientin E.B., ${ }^{*}$ 1927, ältere Dame im Altersheim: Die betagte Dame beklagte sich über Beinbeschwerden. Es wurde eine leichte Arthrose und Thrombophlebitis beidseits (ohne Doppler-Messung) festgestellt. Verschiedene Analgetika brachten keine Besserung. Obwohl die Patientin beschwerdefrei gehen konnte, zog ich eine PAVK in Betracht, da die Dame früher Raucherin war und oft kalte Füsse (aber nicht auch Hände!) hatte und beschloss, eine Therapie mit Padma 28 zu versuchen. In der Folge erhielt die Dame während 2 Monaten dreimal täglich 3 Tabletten (2009 wurde Padma 28 von Tabletten auf Kapseln umgestellt). Nach diesen 2 Monaten hatte die Patientin keine Beschwerden mehr, die Hautläsionen der Thrombophlebitis waren aber noch vorhanden. Zur Sicherheit überwies ich die Dame an einen Angiologen, dessen Urteil positiv war: Die Durchblutung war sehr gut, die Gefässe wiesen keine Schäden auf.

Ich verordnete der Patientin weiterhin zwei- bis dreimal täglich 1 Tablette Padma 28. Nach 2-3 Monaten waren die Hautschäden verschwunden. Die Dame war mit der Behandlung sehr zufrieden, nahm aber weiterhin täglich 1-2 Tabletten ein, obwohl auch die Füsse nicht mehr kalt waren.

Bericht: Dr. med. Pierre-Ami Béguin, Dietikon

Patientin M.M., ${ }^{*} 1921$ :

- Februar 2005: Die Patientin wies an der linken grossen Zehe eine livide Verfärbung mit Rattenbiss-Nekrose auf. Therapie: 2-1-2 Tabletten Padma 28.

- April 2005: Bypass bei PAVK IV, Antikoagulation mit Marcoumar.

- Juli 2005: Der Bypass ist bereits verschlossen, die Zehe leicht gebessert, keine Zunahme der Nekrose.

- Februar 2006: Angiografie: Bypassverschluss, keine Claudicatio, keine Ruheschmerzen.

- Mai 2006: Das subjektive Befinden der Patientin ist viel besser als die Messwerte. Gehtraining, Padma 28 2-0-2.

- Oktober 2008: Die Patientin ist links beschwerdefrei, kann bis zu einer halben Stunde gehen. Keine akralen Läsionen, links stabil, gleichbleibende ABI-Werte.

- Februar 2010: Keine Beschwerden an den Zehen, keine Claudicatio.

- August 2010: Geht bis 1 h. 


\section{Fazit}

2017 feiert das Arzneimittel Padma 28/Padmed Circosan den 40. Jahrestag der Arzneimittelzulassung in der Schweiz, was die erste Registrierung in einem westlichen Land bedeutete. Diese 40 Jahre können als eine

\section{Literatur}

1 Hürlimann F: Eine lamaistische Rezeptformel zur Behandlung der peripheren arteriellen Verschlusskrankheit. Schweiz Rundschau Med (Praxis) 1979;38:1407-1409.

2 Brzosko WJ, et al: Laboratory and clinical studies on Padma 28. Immunol Polska 1983;8:3.

3 Brzosko WJ, et al: Padma 28 a new drug for patients with $\mathrm{HBsAg}$ positive or negative chronic aggressive hepatitis. Hepatol Rapid Lit Rev 1982;8:Memo-H-1971.
Erfolgsgeschichte betrachtet werden, hat sich dieses tibetische Vielstoffgemisch doch in zahllosen Fällen bei Ameisenlaufen, Wadenkrämpfen und Durchblutungsstörungen wie Claudicatio intermittens bewährt. Verschiedene klinische Studien sowie Fallberichte von Ärztinnen und Ärzten be- legen die gute Wirksamkeit und Sicherheit von Padma 28/Padmed Circosan. Somit bietet es sich als Arzneimittel der Wahl bei entsprechenden Beschwerden an.

Ad multos annos!
4 Matzner Y, Sallon S: The effect of Padma 28, a traditional Tibetan herbal preparation, on human neutrophil function. J Clin Lab Immunol 1995;46:13-23.

5 Melzer J, Brignoli R, Diehm C, Reichling J, Do DD, Saller R: Treating intermittent claudication with Tibetan medicine Padma 28: does it work? Atherosclerosis 2006;189:39-46.
6 Vennos C, Melzer J, Saller R: Clinical studies on the efficacy and safety of Padma 28, a complex herbal formulation from Tibetan medicine: an overview. Forsch Komplementmed 2013;20(suppl 2):25-30.

7 Stewart M, Morling JR, Maxwell H: Padma 28 for intermittent claudication. Cochrane Database Syst Rev 2016;(3):CD007371.

8 Ditrych E, Béguin PA: Case Report: Padma 28 bei PAVK - Zwei Fälle aus der Praxis dokumentieren die Wirklichkeit. ARS MEDICI Thema Phytotherapie 2010;10:6-7. 